\title{
Experimental demonstration of spatial quantum correlations in multiple scattering media
}

Smolka, Stephan; Huck, Alexander; Andersen, Ulrik Lund; Lagendijk, Ad; Lodahl, Peter

Published in:

Conference proceedings, CLEO/IQEC

Publication date:

2009

Document Version

Publisher's PDF, also known as Version of record

Link back to DTU Orbit

Citation (APA):

Smolka, S., Huck, A., Andersen, U. L., Lagendijk, A., \& Lodahl, P. (2009). Experimental demonstration of spatial quantum correlations in multiple scattering media. In Conference proceedings, CLEO/IQEC (pp. 1-2). IEEE.

\section{General rights}

Copyright and moral rights for the publications made accessible in the public portal are retained by the authors and/or other copyright owners and it is a condition of accessing publications that users recognise and abide by the legal requirements associated with these rights.

- Users may download and print one copy of any publication from the public portal for the purpose of private study or research.

- You may not further distribute the material or use it for any profit-making activity or commercial gain

- You may freely distribute the URL identifying the publication in the public portal

If you believe that this document breaches copyright please contact us providing details, and we will remove access to the work immediately and investigate your claim. 


\title{
Experimental demonstration of spatial quantum correlations in multiple scattering media
}

\author{
Stephan Smolka ${ }^{1}$, Alexander Huck ${ }^{2}$, Ulrik L. Andersen ${ }^{2}$, \\ Ad Lagendijk ${ }^{3}$, Peter Lodahl ${ }^{1}$ \\ 1. DTU Fotonik, Department of Photonics Engineering, Technical University of Denmark, \\ Building 345V, 2800 Kgs. Lyngby, Denmark \\ 2. DTU Physics, Department of Physics, Technical University of Denmark, Building 309, 2800 \\ Kgs. Lyngby, Denmark \\ 3. FOM Institute for Atomic and Molecular Physics, Kruislaan 407, 1098 SJ Amsterdam, The \\ Netherlands \\ Author e-mail address:stsm@fotonik.dtu.dk
}

\begin{abstract}
We demonstrate that spatial quantum correlations are induced by multiple scattering of squeezed light. The correlation relates multiple scattered photons at different spatial positions, and is tunable by varying photon fluctuations of the illuminating beam.

(C) 2008 Optical Society of America

OCIS codes: (290.4210) Multiple scattering; (270.6570) Squeezed states
\end{abstract}

\section{Introduction}

Multiple wave scattering is an interdisciplinary research field of fundamental and practical importance for nanophotonics, acoustics, and even seismology. Recently, the interest in the quantum nature of multiple scattered light has increased rapidly $[1,2]$, however so far the sparse experiments on quantum noise in multiple scattering media have been in a regime where a phenomenological classical description of classical noise suffices [3, 4]. Here, we present the experimental realization of spatial correlations of photons of genuine quantum origin that are induced by multiple scattering of squeezed light.

An illustration of the spatial quantum correlation is shown in Fig. 1a. The correlation strength is quantified by the spatial quantum correlation function

$$
\overline{C_{a b a b^{\prime}}^{Q}}=\frac{\overline{\left\langle\hat{n}_{a b} \hat{n}_{a b^{\prime}}\right\rangle}}{\overline{\left\langle\hat{n}_{a b}\right\rangle} \times \overline{\left\langle\hat{n}_{a b^{\prime}}\right\rangle}}-1,
$$

where $a$ labels the direction of the incident light and $b, b^{\prime}$ are two different output directions. The operator $\hat{n}_{a b}$ represents the number of photons in output mode $b .\langle\ldots\rangle$ denotes the quantum mechanical expectation value while the bar refers to an average over all realizations of disorder.

a

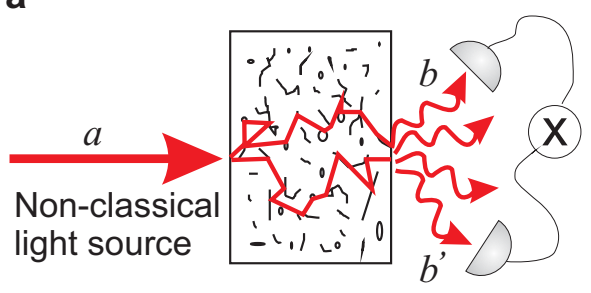

b

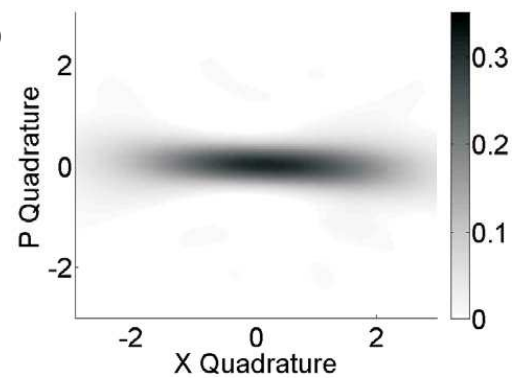

Fig. 1. a, A non-classical light source illuminates a medium consisting of a random distribution of scatterers. The incoming light is split into a multitude of different trajectories that perform a random walk through the medium. The number of photons exiting the medium in a specific direction can be correlated or anti-correlated with the number of photons in another direction, and this spatial quantum correlation depends on the quantum state of light illuminating the medium. b. The reconstructed Wigner function of the measured vacuum squeezing characterizes the non-classicality of the light source. A pronounced reduction (enhancement) in the quantum fluctuations of the $\mathrm{x}(\mathrm{p})$ electric field amplitudes is observed. The Wigner function is normalized to the classical limit. 


\section{JThC5.pdf}

\section{Experiment}
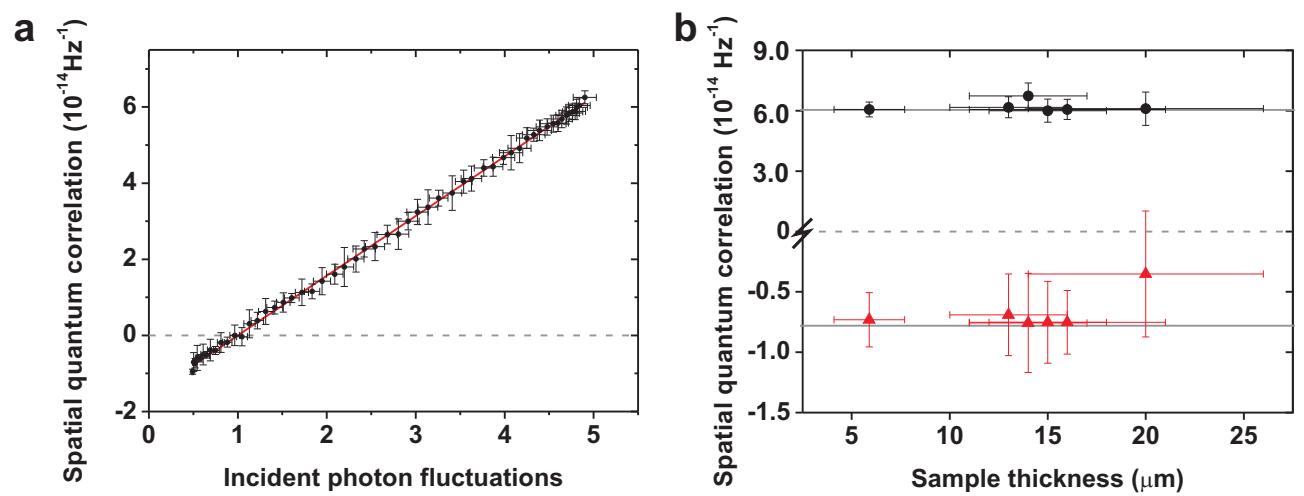

Fig. 2. a, Measured spatial quantum correlation function versus photon fluctuations of the incident light beam relative to the classical level. The data were taken at an input power of $P=120 \mu \mathrm{W}$. For non-classical incident photon fluctuations, negative spatial correlations are observed, while classical incident fluctuations lead to positive spatial correlations. Every data point represents an average over 6 different positions on the sample of thickness $L=6 \mu \mathrm{m}$. The curve is the theoretical prediction without any free parameters and the dashed line represents the uncorrelated case. $\mathbf{b}$, Spatial quantum correlation function versus sample thickness. The spatial quantum correlation function is found to be independent of the sample thickness in agreement with theory (horizontal lines).

As a non-classical source we use vacuum squeezed light generated with a periodically poled KTiOPO4 crystal. The vacuum squeezed state is characterized by the technique of quantum tomography where the Wigner function is reconstructed based on phase-sensitive measurements of the quantum fluctuations (Fig. 1b). We observe that the vacuum squeezed state shows strongly reduced fluctuations in the x electric field amplitude at the expense of enhanced fluctuations in the $\mathrm{p}$ amplitude. The vacuum squeezed state is overlapped with a bright displacement beam on a beam splitter. Depending on the phase difference between the vacuum squeezed state and the displacement beam we are able to tune the photon fluctuations of the light source below and above the classical limit corresponding to a coherent state.

The multiple scattering samples consist of $\mathrm{TiO}_{2}$ particles and the sample thickness is varied between $6 \mu \mathrm{m}$ and $20 \mu \mathrm{m}$. The samples are illuminated with the squeezed light source and the total transmitted photon fluctuations are recorded with a spectrum analyzer. From these measurements the spatial quantum correlation function $\overline{C_{a b a b^{\prime}}^{Q}}$ can be determined [2]. We observe negative (positive) spatial correlations for incident non-classical (classical) fluctuations. The strength of the spatial quantum correlations can be tuned continuously by varying the photon fluctuations of the light source. This pronounced behavior is clearly demonstrated in Fig. 2a and it is in excellent agreement with theory. Furthermore, the spatial correlation function is predicted to be independent of the sample thickness, which holds in the diffusive regime of multiple scattering. This behavior is experimentally confirmed as well, see Fig. $2 \mathrm{~b}$.

\section{Conclusion}

We have presented the first experimental demonstration of spatial quantum correlations that are induced by multiple scattering of squeezed light. The quantum state of our light source was fully characterized by quantum tomography. Depending on the photon fluctuations of the light incident on the sample we demonstrated continuous tuning of the spatial correlation strength. The experimental results were found to be in excellent agreement with the full quantum optics theory for multiple scattered light.

\section{References}

[1] J. Tworzydlo and C.W.J. Beenakker, Phys. Rev. Lett. 89, 043902 (2002).

[2] P. Lodahl, A.P. Mosk, and A. Lagendijk, Phys. Rev. Lett. 95, 173901 (2005).

[3] P. Lodahl and A. Lagendijk, Phys. Rev. Lett. 94, 153905 (2005).

[4] S. Balog, P. Zakharov, F. Scheffold, and S.E. Skipetrov, Phys. Rev. Lett. 97, 103901 (2006). 